\title{
Benchmarking the Enrolment Structure of an Educational System without Exceeding the Carrying Capacity Requirement
}

\author{
Virtue U. Ekhosuehi \\ Department of Mathematics, University of Benin \\ Benin City, Nigeria \\ virtue.ekhosuehi@uniben.edu \\ Augustine A. Osagiede \\ Department of Mathematics, University of Benin \\ Benin City, Nigeria
}

\begin{abstract}
In this paper, we propose an enrolment projection method based on the carrying capacity of the educational system. The method is a refinement of the recruitment control strategy proposed in the literature. We implement our proposed method using enrolment data from a university setting. The results obtained by extrapolating the short-term shifts in enrolment structure reflect the normal progression pattern in the system.
\end{abstract}

Keywords: Carrying capacity, Educational system, Enrolment structure, Markov chain, Recruitment control, Transition model.

\section{Introduction}

In this study, we attempt to answer the question: "What is the future grade structure of the educational system expanding at a uniform rate if present patterns of wastage and promotion continue such that the carrying capacity of the system is not exceeded?" We consider the educational system as a population stratified into $k$ mutually exclusive grades according to various characteristics. The grades in the educational system are nonabsorbing (or transient) states because all movements are towards absorption and away from the grades. Let $S=\{1,2, \cdots, k\}$ be the set of these grades and let $n_{i}(t)$ be the enrolment stock in grade $i$ in session $t$. Then the set of stocks is given by the row vector $\mathbf{n}(t)=\left[n_{1}(t), n_{2}(t), \cdots, n_{k}(t)\right]$. The vector $\mathbf{n}(t)$ is referred to as the structure of the system in session $t$. The session $t$ is a discrete time scale. The flows of students in the system are described by a transition matrix. Moreover, there is a two-way flow between the system and the outside world, viz.: admission flow and wastage flow. Wastage includes dropout and completion (or graduation) states. Dropout encapsulates students who withdraw from the programme before completion. Let the number 0 denote the outside world in which students are transferred. We unify the states of the educational system using Markov chain on the axiomatic foundation that there is a one-stage dependence of events. We focus on the homogeneous case where the probabilities are independent of time. We use the following notations for the transition probabilities of the system:

$\mathbf{P}_{0}=\left\{\left(p_{01}, \cdots, p_{0 k}\right): \sum_{j=1}^{k} p_{0 j}=1, p_{0 j} \geq 0, j \in S\right\}$ is a $1 \times k$ vector of admission probabilities; 


$$
\begin{aligned}
& \mathbf{w}^{\prime}=\left\{\left(p_{10}, \cdots, p_{k 0}\right)^{\prime}: \sum_{i=1}^{k} p_{i 0} \leq 1, p_{i 0} \geq 0, i \in S\right\} \text { is a } k \times 1 \text { vector of wastage probabilities; and } \\
& \mathbf{P}=\left\{\left(p_{i j}\right): \sum_{j=1}^{k} p_{i j} \leq 1, p_{i j} \geq 0, i, j \in S\right\} \text { is a } k \times k \text { transition matrix among the grades. }
\end{aligned}
$$

Since each student in the educational system must either remain in or leave the system, then

$\mathbf{P e}^{\prime}+\mathbf{w}^{\prime}=\mathbf{e}^{\prime}$, where $\mathbf{e}^{\prime}$ is a $k \times 1$ vector of ones. We estimate the elements of $\mathbf{P}_{0}, \mathbf{w}^{\prime}$ and $\mathbf{P}$, from historical data using the maximum likelihood method (Zanakis and Maret, 1980). It is worthy of note that it is rarely feasible to exert direct control over wastage and promotion rates in the educational system. This is because the transitions are largely dependent on students' performance. We therefore adopt recruitment (i.e., admission) control. Specifically, we refine the recruitment control strategy in Bartholomew et al. (1991) to capture the growth rate and the baseline enrolment stock of the system. We define the relative structure of the system, $\mathbf{q}$, over the time interval for which data are available as

$$
\mathbf{q}=\left[\begin{array}{llll}
\sum_{\forall t} n_{1}(t) & \sum_{\forall t} n_{2}(t) & \cdots & \sum_{\forall t} n_{k}(t)
\end{array}\right]\left(\sum_{\forall t} \sum_{i=1}^{k} n_{i}(t)\right)^{-1}
$$

and assume that the system expands at a uniform rate, $g$ (Vassiliou, 1976). We provide estimators for the growth rate and the baseline enrolment stock, which are often unknown to the researcher, via the least squares criterion (Lindgren, 1993). We introduce the carrying capacity of the system so as to ensure that stocks do not exceed the carrying capacity. Thereafter, we formulate a transition model under admission control and then compare the output of our model with the proposals in literature using data from a parttime degree programme in the University of Benin, Nigeria.

\section{Literature review}

The problem of understanding and assessing the flows of students through educational systems has long been dealt with. The works of Gani (1963), Uche (1978), Nicholls (1983, 2009), Osagiede and Ekhosuehi (2006) and Al-Awadhi and Konsowa (2007) are just a few references. In these works, the Markov chain theory is employed. It is worth noting that the common Markov chain formulation for the educational system is derived from the absorbing Markov chain. The basic absorbing Markov chain formulation for the system is represented as: $\mathbf{A}=\left[\begin{array}{ccc}\mathbf{P} & \vdots & \mathbf{w}^{\prime} \\ \ldots & \ldots & \ldots \\ \mathbf{0} & \vdots & \mathbf{I}\end{array}\right]$ (Ekhosuehi and Osagiede, 2012a). The expected time before absorption, denoted as $\mathbf{M S}$, is computed using the formula $\mathbf{M S}=(\mathbf{I}-\mathbf{P})^{-1} \mathbf{e}^{\mathbf{\prime}}$, where the matrix $(\mathbf{I}-\mathbf{P})^{-1}$ is the fundamental matrix of the absorbing Markov chain with its $(i, j)$ th entry being the expected number of times the process is 
in a transient state $i$ before entering an absorbing state $j$ (Ibe, 2009). The transition model arising from the absorbing Markov chain is of the form

$$
\mathbf{n}(t+1)=\mathbf{n}(t) \mathbf{P}+R(t+1) \mathbf{P}_{0}
$$

where $R(t+1)$ is the total number of new entrants into the system in session $t+1$ (Nicholls, 1983).

Osagiede and Ekhosuehi (2006) modeled the educational system using the discrete-time Markov chain (Tsaklidis, 1994) of the form: $\mathbf{Q}=\mathbf{P}+\mathbf{w}^{\mathbf{\prime}} \mathbf{P}_{0}$. The matrix $\mathbf{Q}$ is made up of two matrices: the sub-stochastic matrix $\mathbf{P}$, which corresponds to a direct transition between states, and the replacement matrix $\mathbf{w}^{\prime} \mathbf{P}_{0}$, which is the part of wastage flow that goes back into the system as new entrants. The transition model is of the form

$$
\mathbf{n}(t+1)=\mathbf{n}(t) \mathbf{Q}+g \mathbf{n}(t) \mathbf{I} \mathbf{e}^{\prime} \mathbf{P}_{0} .
$$

The matrix $\mathbf{Q}$ is stochastic as $\mathbf{Q} \mathbf{e}^{\prime}=\mathbf{e}^{\mathbf{\prime}}$. Notably, the projection model (2) can be obtained from equation (1) if we set

$$
R(t+1)=\mathbf{n}(t) \mathbf{w}^{\prime}+g \mathbf{n}(t) \mathbf{I} \mathbf{e}^{\prime} .
$$

Further details on the use of Markov models for students' flows in the educational system are found in Osagiede and Ekhosuehi (2013).

Remarkably, the models in equations (1) and (2) when applied in their raw form for longterm projection give arbitrarily large enrolment stock for the system. Thus, the carrying capacity of the system may be exceeded. This is a snag. The snag can be circumvented by the use of control strategies (Davies, 1982; Kalamatianou, 1987; Bartholomew et al., 1991). In practice, the control problem cannot be completely solved because not all transitions can be controlled, while those that can be controlled are not allowed to be set entirely arbitrarily. This is often the situation in the educational system. Bartholomew et al. (1991) discussed the kinds of strategy for attainability under recruitment control, namely: the fixed strategy and the adaptive strategy. In this case, the number to be recruited $\mathbf{R} *$ in a transition from a current structure $\mathbf{n}$ to a desired structure $\mathbf{n} *$ is

$$
\mathbf{R}^{*}=\left\{\begin{array}{l}
\mathbf{n} *(\mathbf{I}-\mathbf{P}) \text { fixed strategy } \\
\boldsymbol{\delta}=\left(\delta_{i}\right) \text { adaptive strategy }
\end{array},\right.
$$

where $\delta_{i} \leq n_{i}^{*}-X_{i}$ and $X_{i}$ is the $i$ th entry in vector $\mathbf{n P}$.

\section{Materials and methods}

In any planning associated with an expanding educational system, there is a need to be very careful about the impact of the changes in growth rates on the enrolment stocks. For this reason, we modify the model in equation (2) so as to create a bound for the enrolment structure. We achieve this by assuming that management of the educational system will maintain the existing trend in total enrolment size at a point which does not exceed the carrying capacity of each grade of the system and that this maintainability policy will persist into the future. 
Let $N(t)=\mathbf{n}(t) \mathbf{e}^{\prime}$. By assuming that the system expands deterministically at a growth rate of $100 \mathrm{~g} \%$, we have

$$
\Delta N(t)=g N(t) .
$$

Solving the first-order difference equation (5), we obtain the general solution

$$
N(t)=A(1+g)^{t},
$$

where $A$ is an arbitrary constant which is the baseline enrolment stock. We estimate the constants $A$ and $g$ from historical data. Suppose there exist data for sessions $t=1,2, \cdots, T$. Then we transform equation (6) as

$\ln N(t)=\ln A+t \ln (1+g), t=1,2, \cdots, T$.

Taking $\boldsymbol{\Phi}$ and $\mathbf{N}^{\sim}$ respectively as a $T \times 1$ vector of the sessions and as a $T \times 1$ vector with its entry being the natural logarithm of the total enrolment stock in each session, equation (7) becomes

$$
\mathbf{N}^{\sim}=\left[\begin{array}{ll}
\mathbf{e}^{\prime} & \boldsymbol{\Phi}
\end{array}\right]\left[\begin{array}{c}
\ln A \\
\ln (1+g)
\end{array}\right] \text {. }
$$

Solving equation (8), we estimate the growth rate, $g$, as

$$
\hat{g}=\exp \left(\left[\begin{array}{ll}
0 & 1
\end{array}\right]\left(\left[\begin{array}{l}
\mathbf{e}^{\prime} \\
\mathbf{\Phi}
\end{array}\right]\left[\begin{array}{ll}
\mathbf{e}^{\prime} & \mathbf{\Phi}
\end{array}\right]\right)^{-1}\left[\begin{array}{l}
\mathbf{e}^{\prime} \\
\mathbf{\Phi}
\end{array}\right] \mathbf{N}^{\sim}\right)-1,
$$

and the baseline enrolment stock $A$ as

$$
A=\exp \left(\left[\begin{array}{ll}
1 & 0
\end{array}\right]\left(\left[\begin{array}{l}
\mathbf{e}^{\prime} \\
\mathbf{\Phi}
\end{array}\right]\left[\begin{array}{ll}
\mathbf{e}^{\prime} & \mathbf{\Phi}
\end{array}\right]\right)^{-1}\left[\begin{array}{c}
\mathbf{e}^{\prime} \\
\mathbf{\Phi}
\end{array}\right] \mathbf{N}^{\sim}\right)
$$

Substituting equations (9) and (10) into equation (6), we obtain the trend in the total enrolment stock from historical data as

$$
N(t)=(1+\hat{g})^{t} \exp \left(\left[\begin{array}{ll}
1 & 0
\end{array}\right]\left(\left[\begin{array}{l}
\mathbf{e}^{\prime} \\
\mathbf{\Phi}
\end{array}\right]\left[\begin{array}{ll}
\mathbf{e}^{\prime} & \boldsymbol{\Phi}
\end{array}\right]\right)^{-1}\left[\begin{array}{c}
\mathbf{e}^{\prime} \\
\mathbf{\Phi}
\end{array}\right] \mathbf{N}^{\sim}\right)
$$

Thereafter, we construct a transition model to benchmark the expected enrolment stock of the educational system. We achieve this under admission control. We obtain the drift in baseline enrolment structure in terms of the baseline enrolment stock and the relative structure over time. We use the trend formulation in equation (11) to project the total enrolment stock $N\left(\tau_{v}\right), \tau_{v} \in Z_{+}=\{1,2,3, \cdots\}, v=0,1,2, \cdots, \tau_{0} \geq T$. Thus, we obtain the drift in baseline enrolment structure, denoted as $\widetilde{\mathbf{n}}\left(\tau_{v}\right)$, as

$$
\widetilde{\mathbf{n}}\left(\tau_{v}\right)=\mathbf{q}(1+\hat{g})^{\tau_{v}} \exp \left(\left[\begin{array}{ll}
1 & 0
\end{array}\right]\left(\left[\begin{array}{l}
\mathbf{e}^{\prime} \\
\boldsymbol{\Phi}
\end{array}\right]\left[\begin{array}{ll}
\mathbf{e}^{\prime} & \boldsymbol{\Phi}
\end{array}\right]\right)^{-1}\left[\begin{array}{l}
\mathbf{e}^{\prime} \\
\boldsymbol{\Phi}
\end{array}\right] \mathbf{N}^{\sim}\right) \text {. }
$$


Nonetheless, entries in $\widetilde{\mathbf{n}}\left(\tau_{v}\right)$ may exceed the carrying capacity of the system as the model in equation (12) is designed for a system expanding at a uniform rate. We try to circumvent this situation by defining the number of new entrants in such a way that the drift in baseline enrolment structure does not violate the carrying capacity of the system. We therefore have

$$
\mathbf{R}\left(\tau_{v+1}\right)=\min \left(\mathbf{c}\left(\tau_{v+1}\right), \mathbf{q}(1+\hat{g})^{\tau_{v}} \exp \left(\left[\begin{array}{ll}
1 & 0
\end{array}\right]\left(\left[\begin{array}{c}
\mathbf{e}^{\prime} \\
\mathbf{\Phi}
\end{array}\right]\left[\begin{array}{ll}
\mathbf{e}^{\prime} & \boldsymbol{\Phi}
\end{array}\right]\right)^{-1}\left[\begin{array}{c}
\mathbf{e}^{\prime} \\
\mathbf{\Phi}
\end{array}\right] \mathbf{N}^{\sim}\right)\right)(\mathbf{I}-\mathbf{P}) \bullet \mathbf{P}_{0},
$$

where $\mathbf{c}\left(\tau_{v+1}\right)$ is a $1 \times k$ vector which defines the carrying capacity of the system in session $\tau_{v+1}$. The dot $\bullet$ in equation (13) indicates that the multiplication is done elementwise. Thus, $\mathbf{R}\left(\tau_{v+1}\right)$ is a $1 \times k$ vector of new entrants in session $\tau_{v+1}$. Besides, $\mathbf{R}\left(\tau_{v+1}\right)$, $\widetilde{\mathbf{n}}\left(\tau_{v}\right) \in R_{\geq 0}^{k}=R_{\geq 0} \times R_{\geq 0} \times \cdots \times R_{\geq 0}$, where $R_{\geq 0}=\{x \mid x \geq 0\}$. So, we cannot guarantee that the entries in the vectors are integers. For this reason, we use the function ceil(.) to denote the smallest integer greater than or equal to the corresponding elements in a vector. By so doing, we determine the future stocks as

$$
\mathbf{n}\left(\tau_{v+1}\right)=\text { ceil }\left(\mathbf{n}\left(\tau_{v}\right) \mathbf{P}+\mathbf{R}\left(\tau_{v+1}\right)\right)
$$

In the light of the foregoing, our proposal for benchmarking enrolment structure is stated formally as follows:

Let $T: \mathfrak{R} \rightarrow \mathfrak{R}$ be a transformation defined on the product set $\mathfrak{R}=\underbrace{Z_{\geq 0} \times Z_{\geq 0} \times \cdots \times Z_{\geq 0}}_{k \text { times }}$ $\subset R^{k}$, where $R^{k}$ is the $k$-dimensional Euclidean space. Then the expected stock $\mathbf{n}\left(\tau_{v+1}\right)$ is related to $\mathbf{n}\left(\tau_{v}\right) \in \operatorname{dom}(T)$ under admission control as:

$T: \mathbf{n}\left(\tau_{v}\right) \mapsto \operatorname{ceil}\left(\mathbf{n}\left(\tau_{v}\right) \mathbf{P}+\mathbf{R}\left(\tau_{v+1}\right)\right)$,

where

$$
\mathbf{R}\left(\tau_{v+1}\right)=\min \left(\mathbf{c}\left(\tau_{v+1}\right), \mathbf{q}(1+\hat{g})^{\tau_{v}} \exp \left(\left[\begin{array}{ll}
1 & 0
\end{array}\right]\left(\left[\begin{array}{l}
\mathbf{e}^{\prime} \\
\mathbf{\Phi}
\end{array}\right]\left[\begin{array}{ll}
\mathbf{e}^{\prime} & \mathbf{\Phi}
\end{array}\right]\right)^{-1}\left[\begin{array}{l}
\mathbf{e}^{\prime} \\
\mathbf{\Phi}
\end{array}\right] \mathbf{N}^{\sim}\right)\right)(\mathbf{I}-\mathbf{P}) \bullet \mathbf{P}_{0}
$$

Our approach to enrolment projection is relevant in so many ways. Firstly, it provides a technique for computing a uniform growth rate for the system from historical data. Secondly, it incorporates the estimated baseline enrolment stock as well as the growth rate of the system in the admission control model. Thirdly, it accounts for the carrying capacity of the system which is missing in the existing projection models in literature. Finally, unlike the existing methods, the outputs of our approach are integer values. 


\section{Application}

We compare our new model with two models. One, which we denote as Model I, is the projection model in equation (2). The other is a modified version of the model in equation (1) to accommodate the attainability strategy in Bartholomew et al. (1991). We denote the modified version as Model II.

\section{Model II:}

$$
\mathbf{n}\left(\tau_{v+1}\right)=\mathbf{n}\left(\tau_{v}\right) \mathbf{P}+\mathbf{R}\left(\tau_{v+1}\right), \mathbf{R}\left(\tau_{v+1}\right)=\max \left(\mathbf{0}, \mathbf{c}\left(\tau_{v+1}\right)(\mathbf{I}-\mathbf{P}) \bullet \mathbf{P}_{0}\right)
$$

We implement these methods on the enrolment profile of an undergraduate degree programme, B.Sc. Statistics with Computer Science in the University of Benin, Nigeria. The programme has lecture theatres/classrooms with the largest lecture theatre accommodating not more than 1,000 students. The programme is offered in the part-time study mode on a semester basis within a minimum duration of five sessions for students admitted through direct entry into Year 2 and six sessions for those admitted into Year 1. Here, the year of study refers to the grade. New entrants are either admitted into Year 1 or Year 2 of the academic programme. The maximum duration in the programme is a period of nine sessions and this applies only to students admitted into Year 1. A session in the programme is a minimum of nine months. Students in the part-time programme are required to register for a minimum of twenty credits each session out of which they are expected to accumulate a minimum of ten credit passes in order to move to the next higher level. Students who satisfy these requirements are either classified as successful students or students with carry-over courses in the University Senate Format of results. A student considered for graduation must have accumulated not less than 130 credit passes including the General Studies examination. A graduating student who has some outstanding courses is allowed to register and take examinations in those courses at the next available opportunity provided he does not exceed the maximum number of years required for the programme. Students in such a category are classified as referred students. Transitions within the system are hierarchical. Withdrawal from the programme may be due to failure of a student to accumulate up to ten credits in any given session, financial insolvency, death, etc. Such a student is classified under "students who have withdrawn from the university'. Students facing financial and health challenges have the opportunity to apply for temporary withdrawal. If Senate approves any application for temporary withdrawal, then the affected students will be re-instated at a later date into their previous year of study. The event of temporary withdrawal in a given session forms part of the wastage in that session, and the eventual re-instatement into the previous year of study is a part of entry. Nevertheless, the categories of students re-instated are not only those for temporary withdrawal, but also students who were earlier suspended and/or 
rusticated for involving in some irregularities and those whose medical cases were endorsed by the Director of Health Services of the University. Students who miss their examinations are placed in the category "absent from examinations". A student who fails to register for the session is placed in the category "unregistered students". The university policy for the part-time undergraduate course of studies does not allow double promotion, repetition (except in the final year), demotion and transfer, but allows temporary withdrawal. For more information on the regulations and degree requirements for the undergraduate course of study, the Faculty of Physical Sciences Prospectus (2009) of the University is a useful reference book.

To evaluate students' flows in the part-time undergraduate programme, we use enrolment data as contained in Ekhosuehi and Osagiede (2012b) for the period 2003/2004 2008/2009 sessions (Table 1). The figures in parenthesis denote the number of graduates. Looking at the data in Table 1, we find that the flow during the time period $(t-1, t)$ satisfies:

$$
\sum_{j=1}^{k} n_{i j}(t)=n_{i, i+1}(t), n_{i-1, i}(t-1) \geq n_{i, i+1}(t) \text {, for } i \in S \backslash\{2,6\} \text {, and } \sum_{j=1}^{k} n_{j i}(t)=0, \text { for } j>i
$$

In the event of temporary withdrawal and re-instatement, the entry probability vector is the sum of the probability of new entrants and re-instatement of temporary leavers. However, the event of temporary withdrawal is not captured in Table 1 . This may be due to the time-lag between the application for temporary withdrawal and when such an application is approved by Senate. In some cases, the application is done in retrospect, i.e. for a session that has elapsed. If we assume the absence of temporary withdrawal and re-instatement and represent the year of study by the set of levels $S=\{1,2, \ldots, 6\}$, then the absorbing Markov chain $\mathbf{A}$ is 
Virtue U. Ekhosuehi, Augustine A. Osagiede

Table 1: Enrolment data from 2003/2004-2008/2009 at the end of each session

\begin{tabular}{|c|c|c|c|c|c|c|c|c|c|}
\hline \multirow{8}{*}{ 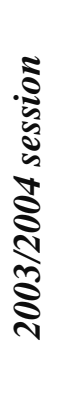 } & $i / j$ & 1 & 2 & 3 & 4 & 5 & 6 & $n_{i 0}$ & $n_{i}(t)$ \\
\hline & $n_{0 j}$ & 112 & 4 & 0 & 0 & 0 & 0 & - & 116 \\
\hline & 1 & 0 & 112 & 0 & 0 & 0 & 0 & 0 & 112 \\
\hline & 2 & 0 & 0 & 53 & 0 & 0 & 0 & 0 & 53 \\
\hline & 3 & 0 & 0 & 0 & 56 & 0 & 0 & 0 & 56 \\
\hline & 4 & 0 & 0 & 0 & 0 & 30 & 0 & 0 & 30 \\
\hline & 5 & 0 & 0 & 0 & 0 & 0 & 35 & 0 & 35 \\
\hline & 6 & 0 & 0 & 0 & 0 & 0 & 8 & $10(10)$ & 18 \\
\hline \multirow{7}{*}{ 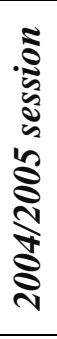 } & $n_{0 j}$ & 110 & - & 0 & 0 & 0 & 0 & - & 110 \\
\hline & 1 & 0 & 106 & 0 & 0 & 0 & 0 & 4 & 110 \\
\hline & 2 & 0 & 0 & 90 & 0 & 0 & 0 & 22 & 112 \\
\hline & 3 & 0 & 0 & 0 & 45 & 0 & 0 & 8 & 53 \\
\hline & 4 & 0 & 0 & 0 & 0 & 48 & 0 & 8 & 56 \\
\hline & 5 & 0 & 0 & 0 & 0 & 0 & 26 & 4 & 30 \\
\hline & 6 & 0 & 0 & 0 & 0 & 0 & 8 & $35(28)$ & 43 \\
\hline \multirow{7}{*}{ 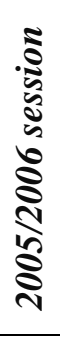 } & $n_{0 j}$ & 236 & - & 0 & 0 & 0 & 0 & - & 236 \\
\hline & 1 & 0 & 234 & 0 & 0 & 0 & 0 & 2 & 236 \\
\hline & 2 & 0 & 0 & 78 & 0 & 0 & 0 & 28 & 106 \\
\hline & 3 & 0 & 0 & 0 & 87 & 0 & 0 & 3 & 90 \\
\hline & 4 & 0 & 0 & 0 & 0 & 45 & 0 & 0 & 45 \\
\hline & 5 & 0 & 0 & 0 & 0 & 0 & 43 & 5 & 48 \\
\hline & 6 & 0 & 0 & 0 & 0 & 0 & 13 & $21(19)$ & 34 \\
\hline \multirow{7}{*}{ 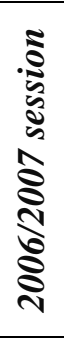 } & $n_{0 j}$ & 353 & - & 0 & 0 & 0 & 0 & - & 353 \\
\hline & 1 & 0 & 346 & 0 & 0 & 0 & 0 & 7 & 353 \\
\hline & 2 & 0 & 0 & 226 & 0 & 0 & 0 & 8 & 234 \\
\hline & 3 & 0 & 0 & 0 & 78 & 0 & 0 & 0 & 78 \\
\hline & 4 & 0 & 0 & 0 & 0 & 87 & 0 & 0 & 87 \\
\hline & 5 & 0 & 0 & 0 & 0 & 0 & 43 & 2 & 45 \\
\hline & 6 & 0 & 0 & 0 & 0 & 0 & 20 & $36(27)$ & 56 \\
\hline \multirow{7}{*}{ 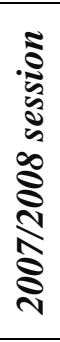 } & $n_{0 j}$ & 471 & 180 & 0 & 0 & 0 & 0 & - & 651 \\
\hline & 1 & 0 & 470 & 0 & 0 & 0 & 0 & 1 & 471 \\
\hline & 2 & 0 & 0 & 404 & 0 & 0 & 0 & 2 & 406 \\
\hline & 3 & 0 & 0 & 0 & 211 & 0 & 0 & 15 & 226 \\
\hline & 4 & 0 & 0 & 0 & 0 & 78 & 0 & 0 & 78 \\
\hline & 5 & 0 & 0 & 0 & 0 & 0 & 80 & 7 & 87 \\
\hline & 6 & 0 & 0 & 0 & 0 & 0 & 35 & $28(27)$ & 63 \\
\hline \multirow{7}{*}{ 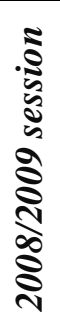 } & $n_{0 j}$ & 181 & 22 & 0 & 0 & 0 & 0 & - & 203 \\
\hline & 1 & 0 & 179 & 0 & 0 & 0 & 0 & 2 & 181 \\
\hline & 2 & 0 & 0 & 489 & 0 & 0 & 0 & 3 & 492 \\
\hline & 3 & 0 & 0 & 0 & 397 & 0 & 0 & 7 & 404 \\
\hline & 4 & 0 & 0 & 0 & 0 & 205 & 0 & 6 & 211 \\
\hline & 5 & 0 & 0 & 0 & 0 & 0 & 67 & 11 & 78 \\
\hline & 6 & 0 & 0 & 0 & 0 & 0 & 44 & $71(65)$ & 115 \\
\hline
\end{tabular}

Source: Ekhosuehi and Osagiede (2012b). 


$$
\begin{gathered}
\text { Nonabsorbing } \\
\text { Nonabsorbing } \\
\text { Absorbin } g
\end{gathered}\left[\begin{array}{cccccc}
p_{11} & p_{12} & \cdots & p_{16} & \vdots & p_{10} \\
p_{21} & p_{22} & \cdots & p_{26} & \vdots & p_{20} \\
\vdots & \vdots & \ddots & \vdots & \vdots & \vdots \\
p_{61} & p_{62} & \cdots & p_{66} & \vdots & p_{60} \\
\cdots & \cdots & \cdots & \cdots & \cdots & \cdots \\
0 & 0 & \ldots & 0 & \vdots & 1
\end{array}\right] .
$$

We estimate the transition probabilities of $\mathbf{A}$ from Table 1 using the maximum likelihood method described in Zanakis and Maret (1980). Thus we obtain

$$
\mathbf{A}=\left[\begin{array}{ccccccc}
0 & 0.9891 & 0 & 0 & 0 & 0 & 0.0109 \\
0 & 0 & 0.9551 & 0 & 0 & 0 & 0.0449 \\
0 & 0 & 0 & 0.9636 & 0 & 0 & 0.0364 \\
0 & 0 & 0 & 0 & 0.9724 & 0 & 0.0276 \\
0 & 0 & 0 & 0 & 0 & 0.9102 & 0.0898 \\
0 & 0 & 0 & 0 & 0 & 0.3891 & 0.6109 \\
0 & 0 & 0 & 0 & 0 & 0 & 1.0000
\end{array}\right] .
$$

Afterward, we estimate the Markov chain $\mathbf{Q}$ from matrix $\mathbf{A}$ as:

$$
\mathbf{Q}=\left[\begin{array}{cccccc}
0.0097 & 0.9903 & 0 & 0 & 0 & 0 \\
0.0398 & 0.0051 & 0.9551 & 0 & 0 & 0 \\
0.0323 & 0.0041 & 0 & 0.9636 & 0 & 0 \\
0.0245 & 0.0031 & 0 & 0 & 0.9724 & 0 \\
0.0797 & 0.0101 & 0 & 0 & 0 & 0.9102 \\
0.5420 & 0.0689 & 0 & 0 & 0 & 0.3891
\end{array}\right]
$$

In matrix $\mathbf{Q}$, the entries in columns 1 and 2 of each transition matrix arise from the replacement matrix and the admission policy of the institution as new entrants are admitted either into Year 1 or Year 2. In particular, the $(1,2)$ entry is the probability that a student is either promoted from Year 1 to Year 2 or the student is admitted into Year 2 to replace leavers in Year 1. Wherever zero entry occurs, it means no transition took place between the corresponding years of study. The main diagonal elements of the transition matrices are either zero or relatively small, while the upper off-diagonal elements ('promotion' probabilities) are large. The main diagonal elements for columns 1 and 2 are small because the wastage probabilities are also very small, while the upper offdiagonal elements are large because a greater proportion of students are promoted. The diagonal entry in column 6 represents the probability of a student repeating Year 6. 
We use equation (9) to estimate the growth rate in the total stock of the academic programme as $\hat{g}=40.56 \%$. We set the carrying capacity for the programme as

$$
\mathbf{c}\left(\tau_{v}\right)=\mathbf{c}=\left[\begin{array}{llllll}
500 & 500 & 500 & 500 & 500 & 800
\end{array}\right], \forall \tau_{v} \in Z_{+}=\{1,2,3, \cdots\} .
$$

Then we compute the expected enrolment structure for a five-year period using Model I, Model II and our new model. The results are presented in Tables $2-4$. We observe that the results in Tables 2 and 3 are not integer values and that most of the results in Table 2 exceed the carrying capacity of the system whereas that of Tables 3 and 4 do not violate the carrying capacity. Tables 3 and 4 also show that the enrolment in each of Year $1-3$ has attained a steady-state in the 2012/2013 session. More so, results in Tables 3 and 4 are approximate except for Year 1 in 2009/2010 session and its subsequent progressions. Thus, our new approach is seen as a refinement of Model II. In sum, the enrolment structure in Table 4 reflects a normal progression in the system.

Table 2: Expected enrolment structure for a five-year period using Model I

\begin{tabular}{|c|c|c|c|c|c|}
\hline 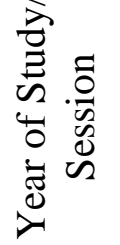 & 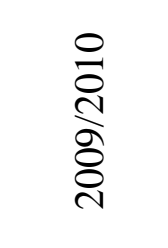 & 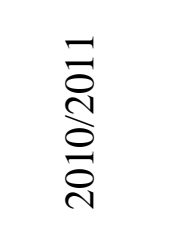 & 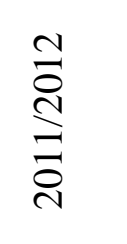 & 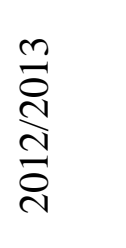 & 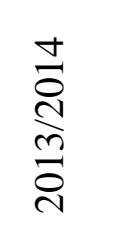 \\
\hline 1 & 641.0625 & 869.4954 & 1266.0 & 1832.6 & 2533.1 \\
\hline 2 & 260.5328 & 744.6239 & 1021.0 & 1485.2 & 2134.6 \\
\hline 3 & 469.9092 & 248.8349 & 711.2 & 975.1 & 1418.5 \\
\hline 4 & 389.2944 & 452.8045 & 239.8 & 685.3 & 939.6 \\
\hline 5 & 205.1764 & 378.5499 & 440.3 & 233.2 & 666.4 \\
\hline 6 & 115.7421 & 231.7868 & 434.7 & 569.9 & 434.0 \\
\hline
\end{tabular}

Source: Author's computation.

Table 3: Expected enrolment structure for a five-year period using Model II

\begin{tabular}{|c|c|c|c|c|c|}
\hline 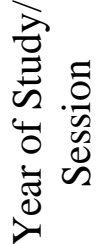 & 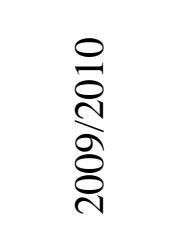 & 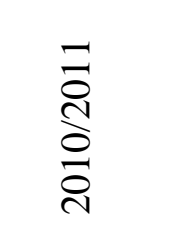 & 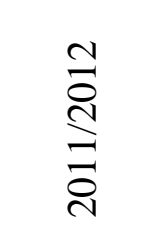 & 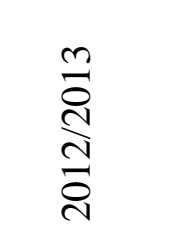 & 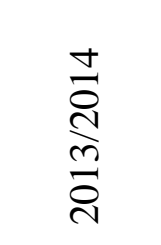 \\
\hline 1 & 443.6000 & 443.6000 & 443.6000 & 443.6000 & 443.6000 \\
\hline 2 & 179.6419 & 439.3795 & 439.3795 & 439.3795 & 439.3795 \\
\hline 3 & 469.9092 & 171.5759 & 419.6514 & 419.6514 & 419.6514 \\
\hline 4 & 389.2944 & 452.8045 & 165.3306 & 404.3761 & 404.3761 \\
\hline 5 & 205.1764 & 378.5499 & 440.3071 & 160.7675 & 393.2153 \\
\hline 6 & 115.7421 & 231.7868 & 434.7443 & 569.9265 & 368.0890 \\
\hline
\end{tabular}

Source: Author's computation. 
Table 4: Expected enrolment structure for a five-year period using new model

\begin{tabular}{|c|c|c|c|c|c|}
\hline 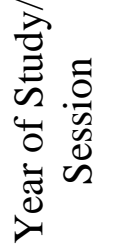 & 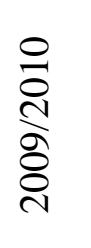 & 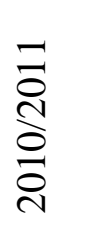 & 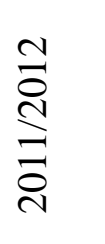 & 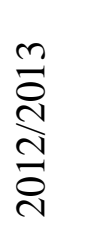 & 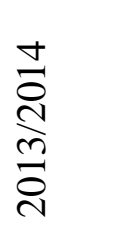 \\
\hline 1 & 431 & 444 & 444 & 444 & 444 \\
\hline 2 & 178 & 427 & 440 & 440 & 440 \\
\hline 3 & 470 & 171 & 408 & 421 & 421 \\
\hline 4 & 390 & 453 & 165 & 394 & 406 \\
\hline 5 & 206 & 380 & 441 & 161 & 384 \\
\hline 6 & 116 & 233 & 437 & 572 & 370 \\
\hline
\end{tabular}

Source: Author's computation.

\section{Conclusion}

In this paper, a new method of enrolment projection based on the carrying capacity of the system was developed. The method is expected to serve as a technical underpinning to benchmarking enrolment stocks in an expanding educational system. The use of our new method is not limited to the educational system alone. It is also applicable to any hierarchical organization where the goal is to attain a desired manpower structure satisfying the capacity requirement. This is because our approach creates a platform wherein the trauma that would have been associated with firing staff numbers equal to the excess capacity requirement is evaded. Exclusively, we have attempted to remedy the challenge emanating from the problem of maintaining a structure at a point which does not exceed the carrying capacity of the educational system. Notwithstanding, it is premature to conclude that our model is the most appropriate mathematical formulation. Further work should be undertaken to identify practical ways to achieve the projected enrolment structure and diffuse possible tensions.

\section{Acknowledgement}

The authors wish to thank the referees for their valuable comments on an earlier version of this paper. We also thank Prof. S. E. Omosigho for pioneering the mathematical aspects of manpower planning in the University of Benin.

\section{References}

1. Al-Awadhi, S. A., and Konsowa, M. (2007). An application of absorbing Markov analysis to the student flow in an academic institution. The $1^{\text {st }}$ Arab Statistical Conference, Arraman-Jordan, pp: 560-580.

2. Bartholomew, D. J., Forbes, A. F., and McClean, S. I. (1991). Statistical techniques for manpower planning ( $2^{\text {nd }}$ ed.). John Wiley \& Sons, Chichester.

3. Davies, G. S. (1982). Control of grade sizes in a partially stochastic Markov manpower model. Journal of Applied Probability, Vol. 19, No. 2, pp: 439-443. 
4. Ekhosuehi, V. U. and Osagiede, A. A. (2012a). On the transition matrix of the flow mechanism in a multi-echelon educational system. International Journal of Operations Research, Vol. 9, No. 4: 209-219.

5. Ekhosuehi, V. U. and Osagiede, A. A. (2012b). The entropy-theoretic stability index for manpower systems. International Journal of Operations Research, Vol. 9, No. 3: 120-128.

6. Faculty of Physical Sciences Prospectus (2009). Prospectus of undergraduate degree (full-time/part-time) \& diploma programmes 2009/2010 session. Faculty of Physical Sciences, University of Benin, Benin City, Nigeria.

7. Gani, J. (1963). Formulae for projecting enrolments and degrees awarded in universities. Journal of the Royal Statistical Society. Series A (General); Vol. 126, No. 3, pp: 400-409.

8. Ibe, C. O. (2009). Markov processes for stochastic modelling. Elsevier Academic Press, Burlington, USA.

9. Kalamatianou, A. G. (1987). Attainable and maintainable structures in Markov manpower systems with pressure in the grades. The Journal of the Operational Research Society, Vol. 38, No. 2, pp: 183-190.

10. Lindgren, B. W. (1993). Statistical theory (4 ${ }^{\text {th }}$ ed.). Chapman \& Hall, New York.

11. Nicholls, M. G. (1983). A Markovian evaluation of a tertiary education faculty. Higher Education 12, pp: 721-730.

12. Nicholls, M. G. (2009). The use of Markov models as an aid to the evaluation, planning and benchmarking of doctoral programs. Journal of the Operational Research Society, Vol. 60, 1183-1190.

13. Osagiede, A. A. and Ekhosuehi, V. U. (2006). Markovian approach to school enrolment projection process. Global Journal of Mathematical Sciences, Vol. 5, No. 1: 1-7.

14. Osagiede, A. A. and Ekhosuehi, V. U. (2013). The use of Markov models in modeling students' flows in a multi-echelon educational system. International Journal of Operational Research, Vol. 17, No. 2: 170 - 182.

15. Tsaklidis, G. M. (1994). The evolution of the attainable structures of a homogeneous Markov system with fixed size. Journal of Applied Probability, Vol. 31, No. 2, pp: 348-361.

16. Uche, P. I. (1978). On stochastic models for educational planning. International Journal of Mathematical Education in Science and Technology, Vol. 9, No. 3: 333-342.

17. Vassiliou, P. C. G. (1976). A Markov chain model for wastage in manpower systems. Operational Research Quarterly (1970-1977), 27(1, Part 1): 57-70.

18. Zanakis, S. H. and Maret, M. W. (1980). A Markov chain application to manpower supply planning. Journal of the Operational Research Society, Vol. 31, pp: 1095-1102. 